PAPER • OPEN ACCESS

Confinement in electron heated plasmas in Wendelstein 7-X and ASDEX Upgrade; the necessity to control turbulent transport

To cite this article: M.N.A. Beurskens et al 2022 Nucl. Fusion 62016015

View the article online for updates and enhancements.
You may also like

- $\frac{\text { Parameter dependences of ion thermal }}{\text { transport due to toroidal ITG turbulence }}$
A.M. Dimits, B.I. Cohen, W.M. Nevins et
al.
- $\frac{\text { Ion temperature profile simulation of JT-60 }}{\text { and TFTR plasmas with ion temperature }}$
$\frac{\text { gradient mode transport models }}{\text { H. Shirai, T. Hirayama, Y. Koide et al. }}$
- The influence of impurities on ion
$\frac{\text { temperature measured by a retarding field }}{\text { analyzer }}$
J Shao, Hai Liu, Yuhong Xu et al.

J Shao, Hai Liu, Yuhong Xu et al. 


\title{
Confinement in electron heated plasmas in Wendelstein 7-X and ASDEX Upgrade; the necessity to control turbulent transport
}

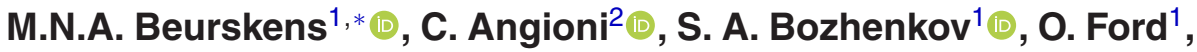 \\ C. Kiefer ${ }^{2}$, P. Xanthopoulos ${ }^{1}{ }^{\circledR}$, Y. Turkin ${ }^{1}$, J.A. Alcusón ${ }^{1}{ }^{\circledR}$, J.P. Baehner ${ }^{1}$, \\ C. Beidler ${ }^{1} \odot$, G. Birkenmeier ${ }^{2}$, E. Fable ${ }^{2}$, G. Fuchert $^{1}$, B. Geiger ${ }^{3}$, \\ O. Grulke ${ }^{1,4}$, M. Hirsch ${ }^{1}$, M. Jakubowski ${ }^{1}{ }^{1}$, H.P. Laqua ${ }^{1}$, A. Langenberg ${ }^{1}\left({ }^{1}\right.$,

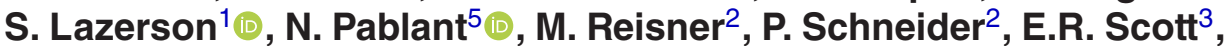 \\ T. Stange ${ }^{1}$, A. von Stechow ${ }^{1}$, J. Stober ${ }^{2}$, U. Stroth ${ }^{2}$, Th. Wegner ${ }^{1}$, G. Weir ${ }^{1}$,

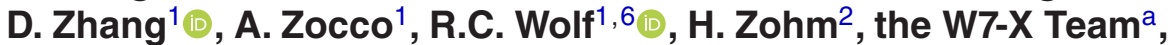 \\ the ASDEX Upgrade Team ${ }^{\mathrm{b}}$ and the EUROfusion MST1 Team $^{\mathrm{c}}$

\footnotetext{
${ }^{1}$ Max-Planck Institut für Plasmaphysik, EURATOM Association, Greifswald, Germany

2 Max-Planck Institut für Plasmaphysik, EURATOM Association, Garching, Germany

${ }^{3}$ University of Wisconsin-Madison, Madison WI, United States of America

4 Technical University of Denmark, Kongens Lyngby, Denmark

5 Princeton Plasma Physics Laboratory, Princeton, NJ, United States of America
} \\ ${ }^{6}$ Technische Universität Berlin, Berlin, Germany \\ E-mail: Marc.Beurskens@ipp.mpg.de
}

Received 31 May 2021, revised 8 October 2021

Accepted for publication 5 November 2021

Published 14 December 2021

\begin{abstract}
In electron (cyclotron) heated plasmas, in both ASDEX Upgrade ( $L$-mode) and Wendelstein $7-\mathrm{X}$, clamping of the ion temperature occurs at $T_{\mathrm{i}} \sim 1.5 \mathrm{keV}$ independent of magnetic configuration. The ions in such plasmas are heated through the energy exchange power as $n_{\mathrm{e}}^{2}\left(T_{\mathrm{e}}-T_{\mathrm{i}}\right) / T_{\mathrm{e}}^{3 / 2}$, which offers a broad ion heating profile, similar to that offered by alpha heating in future thermonuclear fusion reactors. However, the predominant electron heating may put an additional constraint on the ion heat transport, as the ratio $T_{\mathrm{e}} / T_{\mathrm{i}}>1$ can exacerbates ITG/TEM core turbulence. Therefore, in practical terms the strongly 'stiff' core transport translates into $T_{\mathrm{i}}$-clamping in electron heated plasmas. Due to this clamping, electron heated $L$-mode scenarios, with standard gas fueling, in either tokamaks or stellarators may struggle to reach high normalized ion temperature gradients required in a compact fusion reactor. The comparison shows that core heat transport in neoclassically optimized stellarators is driven by the same mechanisms as in tokamaks. The absence of a strong $H$-mode temperature edge pedestal in stellarators, sofar (which, like in tokamaks, could lift the clamped temperature-gradients in the core), puts a strong requirement on reliable and sustainable core turbulence suppression techniques in stellarators.
\end{abstract}

\footnotetext{
* Author to whom any correspondence should be addressed.

a See Klinger et al 2019 (https://doi.org/10.1088/1741-4326/ab03a7) for the W7-X Team.

${ }^{\text {b }}$ See Meyer et al 2019 (https://doi.org/10.1088/1741-4326/ab18b8) for the ASDEX Upgrade Team.

${ }^{c}$ See Labit et al 2019 (https://doi.org/10.1088/1741-4326/ab2211) for the EUROfusion MST1 Team.

(C) (P) Original content from this work may be used under the terms of the Creative Commons Attribution 4.0 licence. Any further distribution of this work must maintain attribution to the author(s) and the title of the work, journal citation and DOI.
} 
Keywords: stellarator transport, turbulent transport, electron heating, ion temperature clamping

(Some figures may appear in colour only in the online journal)

\section{Introduction}

The alpha particles released in the fusion process will predominantly heat the plasmas in a fusion reactor. These alpha particles mostly heat the electrons and at fusion relevant temperatures less than $1 / 3$ of the power goes to the ions. Combined with the direct ion heating, it offers a broad heating profile to the ion through the exchange power. The mostly indirect ion heating may limit the achievable ion temperature. Here we compare two magnetic confinement configuration with similar plasma cross section, but very different magnetic topology and aspect ratio (major radius $R$ over minor radius $a$ ): the tokamak ASDEX Upgrade (AUG, $R=1.65, a=0.52 \mathrm{~m}$ ) and the stellarator Wendelstein 7-X (W7-X, $R=5.5 \mathrm{~m}, a$ $=0.5 \mathrm{~m}$ ). In both devices, plasmas with electron cyclotron resonance heating $(\mathrm{ECRH})$ feature ion temperature clamping where the central ion temperature does not rise above at $T_{\mathrm{i}}$ $\sim 1.5 \mathrm{keV}$ [1]. This clamping is the result of a combination of several effects: (a) the broad ion heating from power transfer from electrons in ECRH heated plasmas depends on plasma density $n_{\mathrm{e}}$ and electron to ion temperature difference as $p_{\mathrm{ex}} \sim n_{\mathrm{e}}^{2} \cdot \frac{\left(T_{\mathrm{e}}-T_{\mathrm{i}}\right)}{T_{\mathrm{e}}^{3 / 2}}$. (b) The increase of heat transport according to the gyro-Bohm scaling where ion heat flux $Q_{\mathrm{i}}$ normalized to the gyroBohm heatflux $Q_{\mathrm{gB}}$ has a strong $T_{\mathrm{i}}$ dependence as $Q_{\mathrm{gB}} \sim n_{\mathrm{i}} T_{\mathrm{i}}^{5 / 2}$. (c) The exacerbated turbulent heat transport due to the effect of increasing $T_{\mathrm{e}} / T_{\mathrm{i}}$ ratio on ion temperature gradient (ITG) or trapped electron mode (TEM) turbulence. This poses a performance limiting challenge for electron heated $L$-mode tokamak plasmas as well as stellarato $r$ plasmas without an edge pedestal or other turbulence reducing features.

The ion heat transport in both devices is dominated by ITG driven turbulence and/or TEM driven turbulence. In a tokamak both turbulence types can occur in presence of low normalized density $(n)$ gradients $\left(a / L_{n}<a / L_{T_{\mathrm{i}}}\right.$, with $\left.L_{x}=x / \nabla x\right)$ and the dominance of turbulence types depends, among other factors, on the collisionality regime, with ITG dominant at high collisionality and TEM being dominant at lower collisionality. In tokamaks, this causes a high degree of ion profile stiffness $[2,3]$, such that their energy confinement is largely set by the achievable edge (ion-) temperature, the so-called pedestal temperature, which lifts up the clamped core temperature (and density). In the $\mathrm{W} 7-\mathrm{X}$ stellarator TEM turbulence is rather benign at low density gradients $\left(a / L_{n}<a / L_{T_{\mathrm{i}}}\right)$ due to its particular 3D geometry [4], but it can still be driven by strong density gradients $\left(a / L_{n}>a / L_{T_{\mathrm{i}}}\right)$. In fact, in conditions where $\left(a / L_{n} \sim a / L_{T_{\mathrm{i}}}\right)$, both ITG and TEM turbulence can be suppressed in W7-X, and be replaced by so-called iTEM turbulence, which drives less transport and may lead to enhance core confinement [5-8], where the (ion) neoclassical transport then becomes a more dominant transport mechanism. These conditions were achieved in W7-X after a train of hydrogen ice pellets produced a peaked density profile. In these plasmas a central ion temperature of more than $T_{\mathrm{i}, 0}=3 \mathrm{keV}$ could be achieved at central density of $n_{\mathrm{e}, 0}=8 \times 10^{19} \mathrm{~m}^{-3}$, with hydrogen as a fuel and with only $5 \mathrm{MW}$ of ECRH [9-11]. However this enhanced performance was so-far only achieved transiently for as long as the high core density gradients were sustained $(\sim 100 \mathrm{~ms})$.

In comparison, stationary gas-fueled ECRH plasmas in W7-X feature flat to slightly peaked density profiles with $a / L_{n}<a / L_{T_{\mathrm{i}}}$. Under such conditions the ion temperature is clamped at $T_{\mathrm{i}} \sim 1.5 \mathrm{keV}$ (figure 1), despite a wide variation of central ECRH input power and electron density (see figure caption). Counter to the post-pellet plasmas in [9] where $T_{\mathrm{i}}>$ $3 \mathrm{keV}$ was transiently achieved, the clamping of $T_{\mathrm{i}}$ appears virtually irrespective of applied ECRH power and obtained density and electron temperature values. Figure $1(a)$ shows that in W7-X virtually the same $T_{\mathrm{i}} \sim 1.5 \pm 0.2 \mathrm{keV}$ is achieved in four configurations with various degree of neoclassical optimization. This observation was puzzling at first, as one would expect a clear difference in performance on the basis of neoclassical simulations between low $\varepsilon_{\text {eff }} \sim 0.8 \%$ and high $\varepsilon_{\text {eff }} \sim$ $2.5 \%$ configurations, as shown by respectively the gray triangles and gray diamonds in the figure. The neoclassical transport simulations were obtained with the neoclassical transport solver suite (NTSS) using the mono-energetic transport coefficients for both configurations as calculated using the drift kinetic equation solver (DKES) [12,13]. For the simulations flat density profiles spanning a wide range of densities and a for W7-X median centrally deposited $P_{\mathrm{ECRH}}=4.5 \mathrm{MW}$ is assumed. It should be noted that beside the configuration insensitivity, in the experiments the clamped central $T_{\mathrm{i}}$ lies well below the neoclassical predictions, which offers further indication that turbulent transport is at play.

In a direct comparison to W7-X, an AUG experiment was conducted in hydrogen gas-fueled plasmas with 99 different ECRH-power and density combinations $\left(P_{\mathrm{ECRH}}=0.5-5 \mathrm{MW}\right.$ and $\left.n_{\mathrm{e}, 0}=2-8 \times 10^{19} \mathrm{~m}^{-3}\right)$. The studied database consists of stationary plasma phases in divertor configuration with a lower single null. The discharges were performed in the favorable magnetic configuration for $L-H$ transitions with the grad$\mathrm{B}$ drift and the curvature drift pointing toward the $X$-point. The elongation was in the range of $\kappa=1.5-1.7 . B_{\mathrm{t}}=2.5 \mathrm{~T}$ and $I_{\mathrm{p}}=1.0-1.2 \mathrm{MA}$, leading to an edge safety factor of $q_{95} \approx 3.7-4.4$. Figure $1(b)$ shows central $T_{\mathrm{i}}$ versus $T_{\text {e. }}$ On average, the ion temperature is $T_{\mathrm{i}}=1.2 \mathrm{keV}$ and shows little variation with ECRH power level and plasmas density (for the 

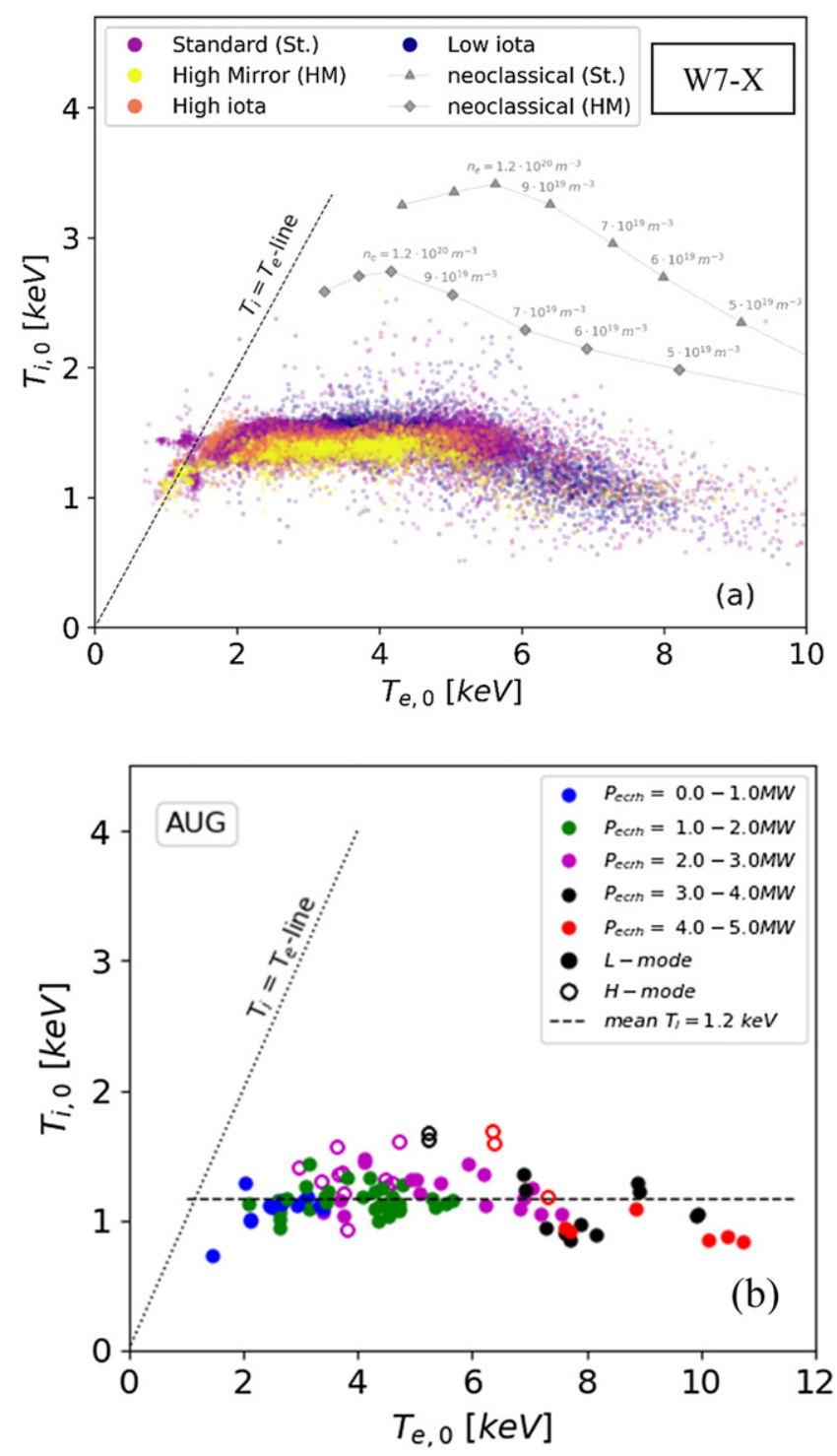

Figure 1. Core ion temperature versus electron temperature in ECRH heated hydrogen gas fueled plasmas in $(a)$ W7-X with a variation of $P_{\mathrm{ECRH}}=1-7 \mathrm{MW}$ and $n_{\mathrm{e}}=0.2-1.4 \times 10^{20} \mathrm{~m}^{-3}$ for four magnetic configurations. Also shown in gray triangles and diamonds are neoclassical simulation for respectively the 'standard' $\varepsilon_{\text {eff }} \sim 0.8 \%$ and the 'high mirror' $\varepsilon_{\text {eff }} \sim 2.5 \%$ configuration. In the simulations, the standard configuration would reach the highest ion temperatures thanks to its better neoclassical optimization and smaller effective magnetic ripple $\left\langle\varepsilon_{\text {eff }}\right\rangle$. (b) AUG with a variation of $P_{\mathrm{ECRH}}=1-5 \mathrm{MW}$ and $n_{\mathrm{e}, 0}=2-8 \times 10^{19} \mathrm{~m}^{-3}$, showing $L$-mode plasmas in full and $H$-mode plasmas in open symbols. The colored points are grouped per $1 \mathrm{MW}$ power step.

latter refer to figure $2(a)$ ). Also, the confinement state is indicated with $L$-mode given by full, and $H$-mode confinement by open symbols, respectively. The $H$-mode plasmas have been identified using an edge localized mode signature in the divertor $\mathrm{H}_{\alpha}$ emission. Figure 2(a) shows that plasmas with high ECRH power and/or high density have undergone an $L-H$ transition, consistent with the density dependence of the $L-H$ power threshold as in e.g. [14]. Many of those have developed an edge transport barrier in both temperature and density profile, which lifts up the core profiles and results in higher central
$T_{\mathrm{i}}$ values. Figure $2(b)$ shows the strong coupling of the edge and core $T_{\mathrm{i}}$ in the data set at hand.

In this paper we compare the core ion heat transport in centrally heated ECRH plasmas between AUG and W7-X and verify that indeed the combination of the following contribute to the clamping of the ion temperature. We investigate: (1) the broad indirect ion heating by the power exchange profile, (2) the strong ion temperature dependence of the microturbulence related gyroBohm transport and (3) the exacerbation of the (ITG and TEM) turbulent transport due to an increased ratio of $T_{\mathrm{e}} / T_{\mathrm{i}}$ in ECRH plasmas. Mitigation the effect of the enhanced turbulent transport is also discussed and is found either by providing an $\mathrm{H}$-mode edge barrier or by core turbulence mitigating techniques.

\section{Ion-heating and transport in electron-heated plasmas}

In ECRH (and other electron-heated) plasmas, the ions are heated through equipartition of the hot electrons with the cold ions. Following [15], the exchange power density is estimated for a single-species plasma as:

$$
p_{\mathrm{ex}}=38 \cdot n_{\mathrm{e}}^{2} \cdot \frac{\left(T_{\mathrm{e}}-T_{\mathrm{i}}\right)}{T_{\mathrm{e}}^{3 / 2}} \cdot \frac{Z}{M}\left[\mathrm{KW} \mathrm{m}^{-3}\right],
$$

where $Z$ is the species charge and $M$ the species mass number. The local ion heatflux $Q_{\mathrm{i}}$ in electron heated plasmas, is calculated as the volume integral of (1). For typical temperature profiles, this leads to a broad ion heating profile. In addition, the micro turbulence driven gyroBohm heat flux has a strong $T_{\mathrm{i}}$ dependence as e.g. in [16]:

$$
Q_{\mathrm{gB}}=\sqrt{\frac{m_{\mathrm{i}}}{e}} \frac{n_{\mathrm{i}} T_{\mathrm{i}}^{5 / 2}}{a^{2} B^{2}},
$$

where $m_{\mathrm{i}}$ is the ion mass, e the electron charge, $n_{\mathrm{i}}$ the ion density and $B$ the magnetic field. In micro turbulence theory the effect of e.g. ITG and TEM on transport is given by the ratio of $Q_{\mathrm{i}}$ and $Q_{\mathrm{gB}}$ which has a strong ion temperature dependence as $Q_{\mathrm{i}} / Q_{\mathrm{gB}} \sim n_{\mathrm{e}} \cdot T_{\mathrm{i}}{ }^{-5 / 2}$, implying that the turbulence is greatly enhanced by an increase in $T_{\mathrm{i}}$ itself, on top of any additional gradient limiting effects.

In addition, other factors can affect the level of turbulent transport. For example in [9-11] the density gradient has a mitigating effect on the micro turbulence, and in W7$\mathrm{X}$ experiments it was found that when $a / L_{n_{\mathrm{e}}}$ is increased and $a / L_{n_{\mathrm{e}}}=a / L_{T_{\mathrm{i}}}$ align ITG and TEM turbulence can be suppressed simultaneously [8]. On the other hand, ITG turbulence can be greatly exacerbated with increasing ratio of $T_{\mathrm{e}} / T_{\mathrm{i}}$ as has been discussed in [17] for both tokamaks and stellarator. Particularly for ECRH plasmas this is potentially an important factor as typically the electron heating leads to $T_{\mathrm{e}} / T_{\mathrm{i}}>1$.

\subsection{ECRH power scan experiments in ASDEX Upgrade}

In an attempt to demonstrate these effects, a dedicated AUG experiment is conducted with several ECRH power scans from 

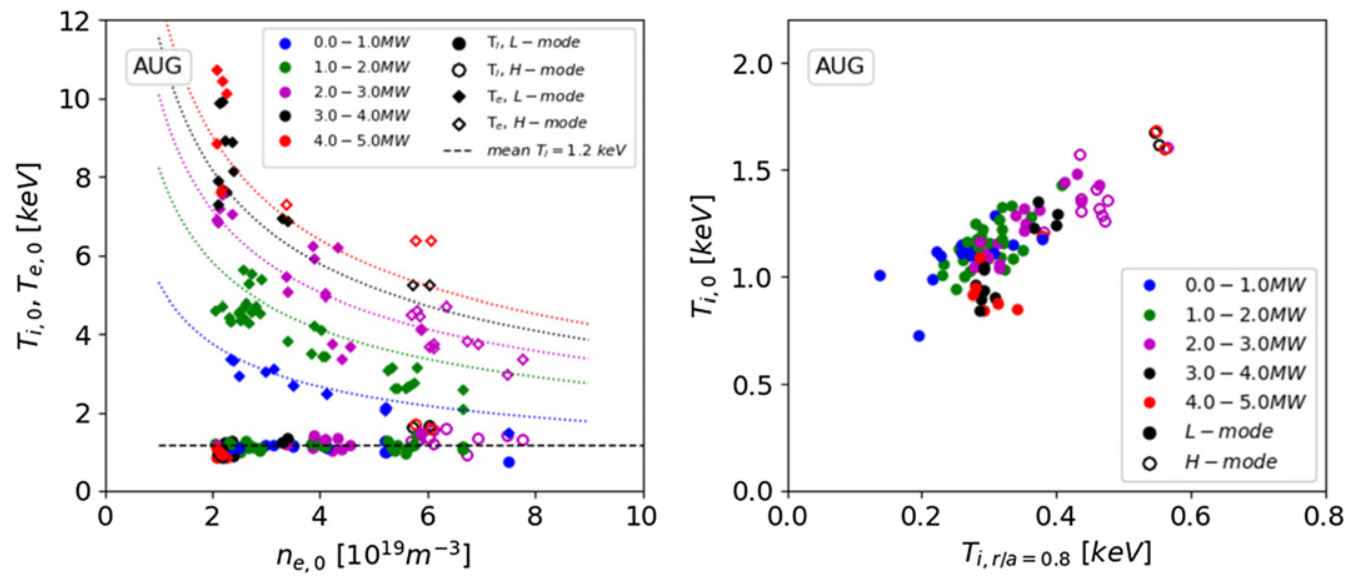

Figure 2. (a) AUG central $T_{\mathrm{i}, 0}$ and $T_{\mathrm{e}, 0}$ versus $n_{\mathrm{e}, 0}$ for the same plasmas as in figure $1(b)$, showing that $T_{\mathrm{i}, 0}$ is virtually independent of $T_{\mathrm{e}, 0}$ and $n_{\mathrm{e}, 0} . L-H$ mode transitions are observed at the higher end of ECRH power and/or higher plasma density in the scans, i.e. consistent with the $H$-mode power threshold for low and medium density in the literature. (b) Coupling of core $T_{\mathrm{i}}$ with edge $T_{\mathrm{i}}$, demonstrating the benefit of an $H$-mode barrier on core confinement.

$P_{\mathrm{ECRH}}=0-4 \mathrm{MW}$ in hydrogen plasmas at densities of subsequently $n_{\mathrm{e}, 0}=2 \times 10^{19}$ and $3 \times 10^{19} \mathrm{~m}^{-3}, B_{\mathrm{t}}=2.5 \mathrm{~T}$ and $I_{\mathrm{p}}=1.2 \mathrm{MA}$, leading to an edge safety factor of $q_{95} \approx 3.7$. At these low densities, the plasmas stay in $L$-mode confinement. Figures 3(b)-(d) shows the profiles of $T_{\mathrm{e}}, T_{\mathrm{i}}$ and $n_{\mathrm{e}}$ obtained in these ECRH power scans. The density profiles are flat and $a / L_{n_{\mathrm{e}}}$ is therefore low, whereas $a / L_{T_{\mathrm{i}}}$ remains unchanged in the power scan across the entire plasmas crosssection (figure 3(e)) with $a / L_{T_{\mathrm{i}}}>a / L_{n_{\mathrm{e}}}$. In absence of an edge pedestal in these $L$-mode plasmas, the ion temperature profile remains virtually unchanged across the variation of $A \cdot Q_{\mathrm{i}}$ (figure $3(f)$ ), where $A$ is the flux surface area.

In order to demonstrate the effect of an $L-H$ transition on the core profiles, we select a deuterium plasma with a strong $H$-mode pedestal after the transition. In the deuterium $L$-mode plasmas, the ion temperature and its normalized gradients (figures $4(d)$ and $(e)$ ) are clamped at similar levels as those in the hydrogen plasmas, despite the lower ion heat flux in deuterium (figures $3(f)$ versus $4(f)$ ) due to the inverse mass dependence of the energy exchange term [15]. On the other hand, due to the isotope dependence of the $L$ to $H$ transition, the deuterium plasmas undergo a transition to $H$-mode, at lower input power than the hydrogen plasmas (figure 4(a) open and closed symbols). After the transition, an edge transport barrier produces a pedestal in $T_{\mathrm{e}}, T_{\mathrm{i}}$ and $n_{\mathrm{e}}$ for $r / a>$ 0.9 (figures $4(b)-(d)$ ). Thanks to the $T_{\mathrm{i}}$ pedestal, the core ion temperature profile can be lifted and the core $T_{\mathrm{i}}$ clamping be broken (figure 4(c)). Nevertheless, the normalized ion temperature gradient is not enhanced (figure $4(e)$ ) and remains at or below $a / L_{T_{\mathrm{i}}}<2$ at mid radius $r / a \sim 0.5$, as was found for the $\mathrm{H}_{2}$ and $\mathrm{D}_{2} L$-mode plasmas.

We show the local gradient changes for the radius $r / a=0.5$ (figure 5) to illuminate the mechanisms at hand. As the ECRH power increases in the hydrogen plasmas, the ratio of $T_{\mathrm{e}} / T_{\mathrm{i}}$ (figure $5(a)$ ) increases strongly but the increase of $Q_{\mathrm{i}}$ (figure 5(b)) is only modest for the higher density plasmas and null for the lower density. This is due to the strong $T_{\mathrm{e}}^{-3 / 2}$ dependence in (1). Moreover, the ratio of $Q_{\mathrm{i}} / Q_{\mathrm{gB}}$ (figure $5(c)$ ), relevant for micro turbulence, is even stronger suppressed due to the $T_{\mathrm{i}}^{5 / 2}$ dependence in equation (2). Therefore, the increase of the electron to ion exchange power in AUG ECRH plasmas is too weak to be able to compete with the increasing micro turbulence.

The deuterium ECRH plasmas have similar issues in raising the local gradients. After the $L-H$ transition the core $T_{\mathrm{i}}$ value increases proportionally with the pedestal $T_{\mathrm{i}}$. However, the normalized $T_{\mathrm{i}}$ gradients $a / L_{T_{\mathrm{i}}}$ remain the same or even decrease (figure $4(e)$ ). Figure 6 shows that the ratio of $T_{\mathrm{e}} / T_{\mathrm{i}}$ does not vary strongly in the ECRH scan due to the $L-H$ transition and accompanying density increase. Therefore the increase of $Q_{\mathrm{i}}$ with $P_{\mathrm{ECRH}}$ is modest and $Q_{\mathrm{i}} / Q_{\mathrm{gB}}$ even decreases after the $L-H$ transition due to the $T_{\mathrm{i}}^{5 / 2}$ dependence in $Q_{\mathrm{gB}}$. So it appears that in both the $\mathrm{H}_{2}$ and $\mathrm{D}_{2}$ plasma examples presented here, the exchange power is too weak to provide a strong enough drive for $a / L_{T_{\mathrm{i}}}$ in an ECRH scan. In experiments in which the ion heat flux can be significantly increased through the application of auxiliary ion heating by NBI, one observes that larger changes of $a / L_{T_{\mathrm{i}}}$ are possible, particularly at low values of $T_{\mathrm{e}} / T_{\mathrm{i}}$ [3].

Predictive runs of these plasmas have been performed with the ASTRA transport code [18] and the TGLF quasi-linear turbulent transport model [19]. Detailed description of the simulations here can be found in [20]. The quasi-linear transport model TGLF is used with the saturation rule SAT1geo, which includes the geometry dependence of the fluctuation intensity [21]. To avoid the use of a predictive pedestal model, the boundary conditions for the ASTRA-TGLF simulations are taken from the experiment with $T_{\mathrm{i}}, T_{\mathrm{e}}, n_{\mathrm{e}}$ at $r / a \sim 0.84$, which is inside the pedestal region. Firstly the simulations identify the dominant micro instability (the instability with the highest $\gamma / k_{\perp} \rho$, where $\gamma$ is the growth rate, $k_{\perp}$ is the poloidal wavenumber of the considered mode and $\rho$ is the sound ion gyro radius). ITG turbulence is most dominant at high collisionality $\left(\sim n_{\mathrm{e}} / T_{\mathrm{e}}^{2}\right)$ and TEM at low collisionality (see [20] for full analysis). Figure 7( $a$ ) shows that at $r / a=0.5$, the low density hydrogen plasmas are largely TEM dominated whereas 

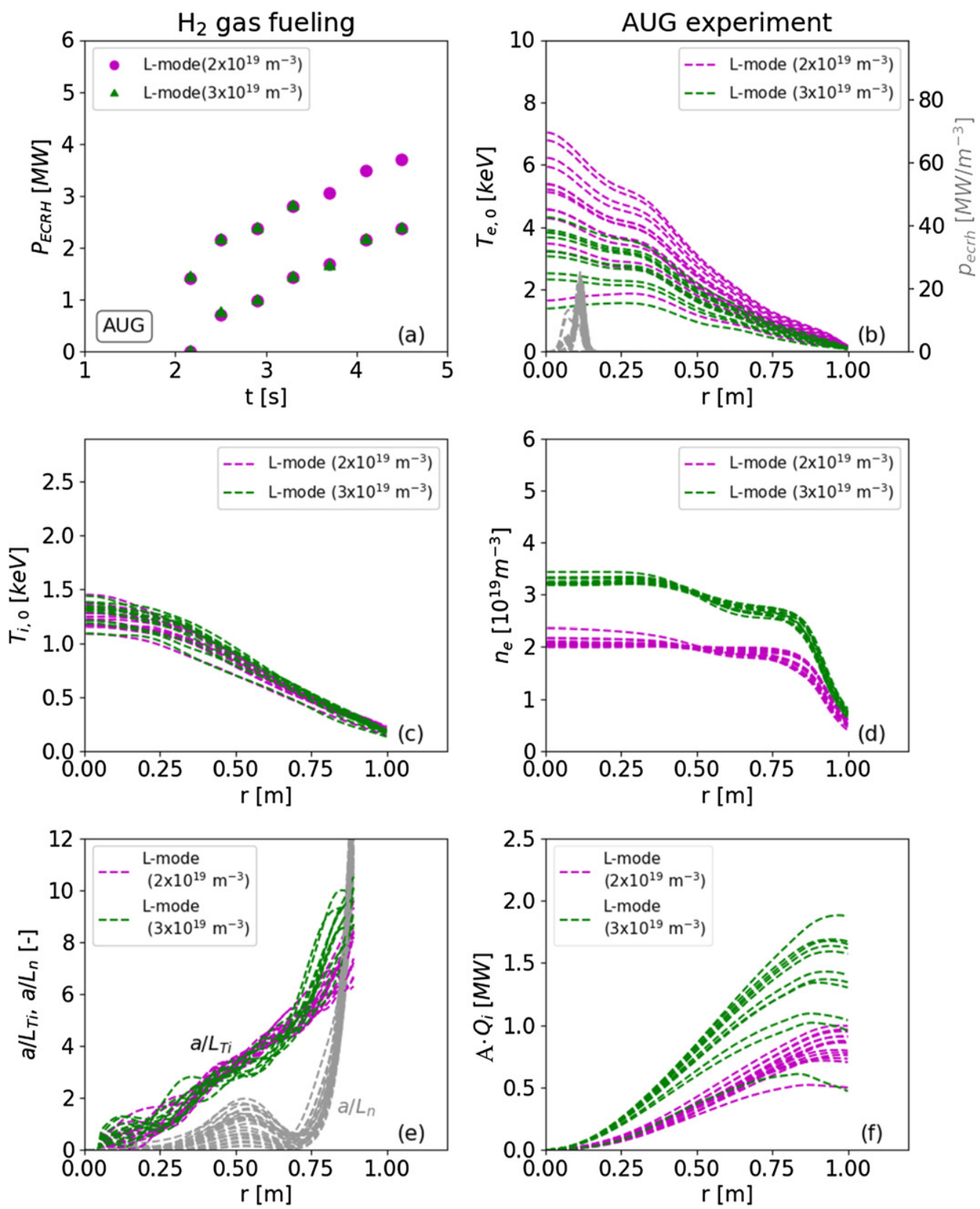

Figure 3. AUG: ECRH power scan from $0-4 \mathrm{MW}$ in $L$-mode hydrogen fueled plasmas for two density levels (purple and green) showing: (a) ECRH power levels. (b) Electron temperature $T_{\mathrm{e}}$ and ECRH deposition profiles. (c) Clamped ion temperature $T_{\mathrm{i}}$ profiles. (d) Electron density profiles. (e) Inversed gradient lengths $a_{\mathrm{eff}} / L_{T_{\mathrm{i}}}$ (purple and green) and $a_{\mathrm{eff}} / L_{n}$ (gray) using $a_{\mathrm{eff}}=0.6 \mathrm{~m}$ for AUG. $(f)$ Ion heat flux obtained from the electron to ion exchange power with $A$ the flux surface area. (Pulse \#38174 and \#38175 for low density and \#38176 and \#38177 for high density.)

the medium density hydrogen plasmas transition from TEM to ITG at the lower ECRH powers, but remain TEM dominated at large $P_{\mathrm{ECRH}}>1.5 \mathrm{MW}$. The collisionality dependence means that at larger radii ITGs become more dominant and vice versa at small radii TEMs play a larger role. For the deuterium plasmas, a similar observation is made in figure $7(b)$. The low-density plasma (incl the $H$-mode phase) is largely TEM dominated whereas the medium density plasma is largely ITG dominated.
The ratio of $T_{\mathrm{e}} / T_{\mathrm{i}}$ can exacerbate ITG [17] and possibly TEM [22] turbulence and hence the ion heat transport. A clear demonstration of this mechanism was given in [3], figures 5 and 6 therein, where the degree of profiles stiffness was greatly enhanced when the ratio of $T_{\mathrm{e}} / T_{\mathrm{i}}$ was increased from 1.5 to 2. In that experiment the ion heat flux was varied by means of on and off-axis neutral beam heating (NBI) as well as central ECRH to induce a variation of $Q_{\mathrm{i}} / Q_{\mathrm{gB}}$. However, in the pure ECRH experiments presented in the current study, 

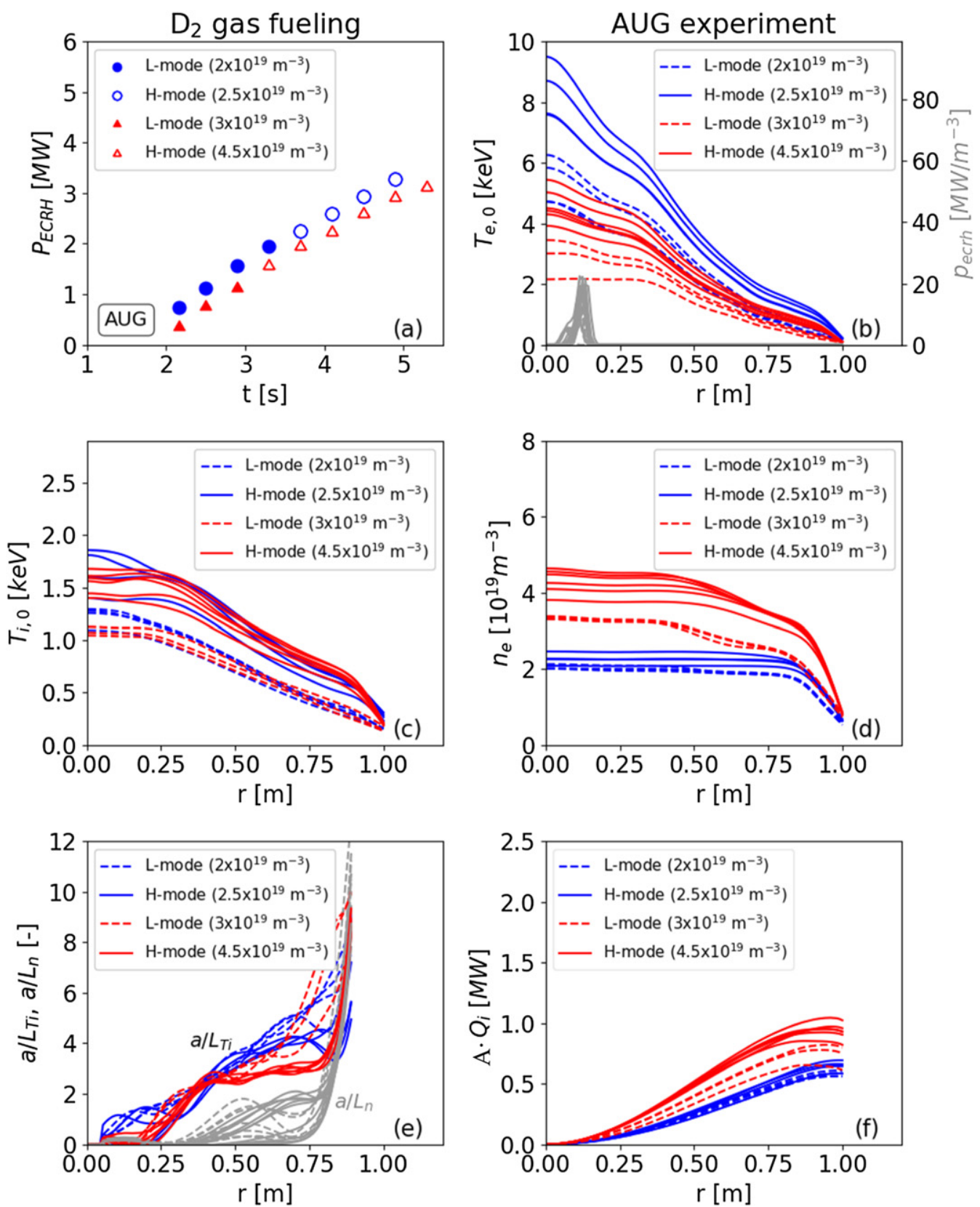

Figure 4. AUG: ECRH power scan from 0-3.5 MW in deuterium fueled plasmas for two different density levels (red and blue). (a) ECRH power levels: at a certain threshold power level the deuterium plasmas feature an $L-H$ mode transition, indicated by open $(L$-mode) and closed symbols $(H$-mode) in this figure and by dashed $(L$-mode) or drawn lines (H-mode) in other figures $(b)-(f)$. $(b)$ Profiles of electron temperature $T_{\mathrm{e}}$ and ECRH deposition profile, $c$ ) ion temperature $T_{\mathrm{i}},(d)$ electron density, $(e)$ inversed gradient lengths $a_{\mathrm{eff}} / L_{T_{\mathrm{i}}}$ (blue and red) and $a_{\text {eff }} / L_{n}$ (gray) with $a_{\text {eff }}=0.6 \mathrm{~m},(f)$ ion heat flux obtained from the electron to ion exchange power, with A the flux surface area. (Pulse \#37908 for low density and \#37909 for high density.)

the strength of the $T_{\mathrm{e}} / T_{\mathrm{i}}$ turbulent transport drive cannot be tested, as ion heat flux $Q_{\mathrm{i}} / Q_{\mathrm{gB}}$ saturates or even declines in the $\mathrm{H}_{2}$ and $\mathrm{D}_{2}$ ECRH power scans (figures $5(c)$ and 6(c)). However, the clamping of the ion temperature, or better of the normalized $T_{\mathrm{i}}$ gradient in the AUG ECRH heated plasmas at $a / L_{T_{\mathrm{i}}}{ }^{\mathrm{ECRH}-\exp } \sim 2$ is shown in figure $8(a)$ for $r / a=0.5$, and is reproduced by the ASTRA-TGFL simulations with $a / L_{T_{\mathrm{i}}}$, ,STRA-TGLF $\sim 2$ in figure $8(b)$ (with $a=a_{\mathrm{eff}}=0.6$ for AUG). Across the plasmas cross section, i.e. from the sawtooth inversion radius $r / a \sim 0.3$ toward the edge $r / a \sim 0.7$, the data show similar clamping as in figure 8, and similar good reproduction of this clamping by the ASTRA-TGLF code. However, TGLF is additionally restricting $a / L_{T_{\mathrm{i}}}$ when $T_{\mathrm{e}} / T_{\mathrm{i}}>3$.

The $T_{\mathrm{i}}$ (gradient) clamping observed in the ECRH plasmas in AUG is therefore likely caused by a combination of the weak exchange power with a broad deposition profile, as well as gyroBohm-like ion heat transport suppressing $Q_{\mathrm{i}} / Q_{\mathrm{gB}}$. Conclusively, the ratio of $T_{\mathrm{e}} / T_{\mathrm{i}}$ may also play a role on the $T_{\mathrm{i}^{-}}$ clamping in these plasmas, but this could not be verified in the 

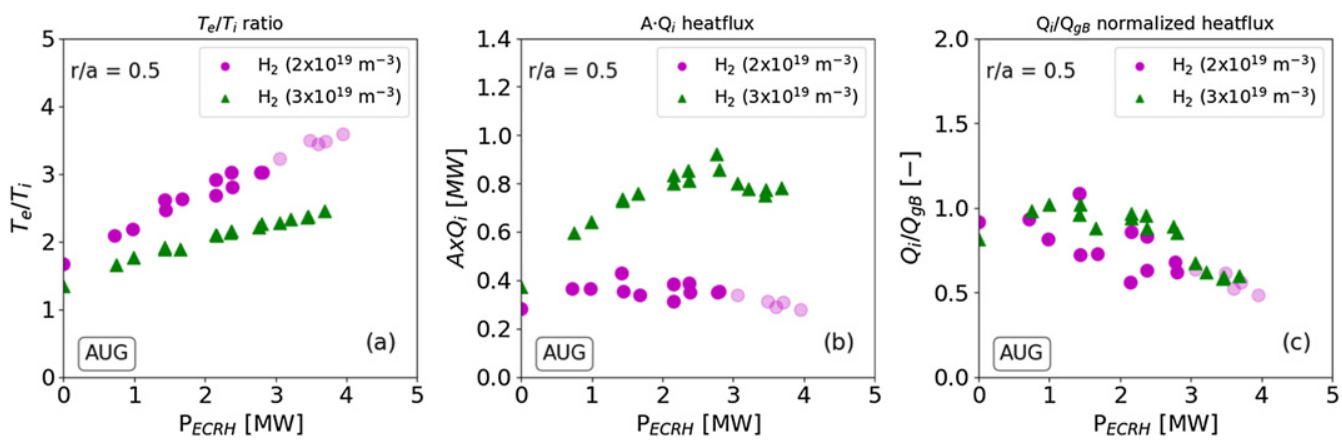

Figure 5. AUG: $(a)$ the ratio of $T_{\mathrm{e}} / T_{\mathrm{i}}(b)$ ion heatflux $A \cdot Q_{\mathrm{i}}$, and $(c)$ the ratio of $Q_{\mathrm{i}} / Q_{\mathrm{gB}}$ as a function of $P_{\mathrm{ECRH}}$ at radius $r / a=0.5$ for hydrogen plasmas. Points with $T_{\mathrm{e}} / T_{\mathrm{i}}>3$ are marked for the simulations in figure 8 .
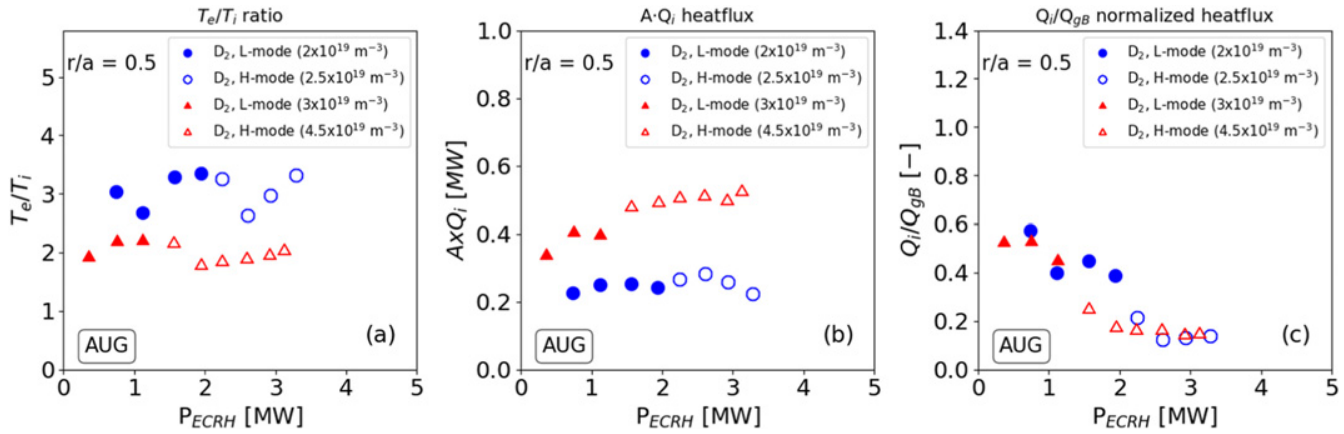

Figure 6. AUG: (a) the ratio of $\left.T_{\mathrm{e}} / T_{\mathrm{i}} b\right)$ ion heatflux $A \cdot Q_{\mathrm{i}}$, and $(c)$ the ratio of $Q_{\mathrm{i}} / Q_{\mathrm{gB}}$ as a function of $P_{\mathrm{ECRH}}$ at radius $r / a=0.5$ for deuterium plasmas.
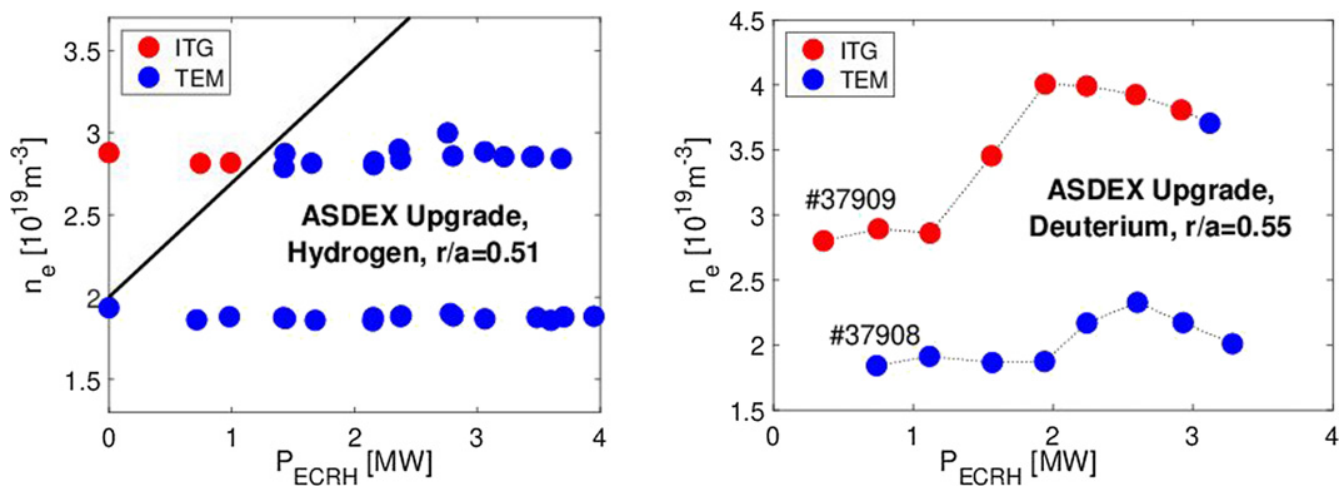

Figure 7. AUG: most dominant ion scale instability for the hydrogen plasmas (left) and deuterium plasmas (right) for $r / a=0.5$. ITG turbulence is most dominant at high collisionality $\left(\sim n_{\mathrm{e}} / T_{\mathrm{e}}{ }^{2}\right)$ and TEM at low collisionality. For ITG, the real frequency is $\omega_{\mathrm{r}}<0$ and for TEM $\omega_{\mathrm{r}}>0$ in TGLF convention.

experiments presented here. However, A negative impact of increasing $T_{\mathrm{e}} / T_{\mathrm{i}}$ has been demonstrated with mixed NBI and ECRH heating in AUG $H$-mode plasmas in [3].

\subsection{ECRH power scan experiment in $W 7-X$}

In W7-X, a power scan from $P_{\mathrm{ECRH}}=1.2 \mathrm{MW}$ to 3.9 MW is conducted in the 'standard' magnetic configuration $\left(\left\langle\varepsilon_{\text {eff }}\right\rangle=0.8 \%\right.$ and $\left.\iota=5 / 5\right)$ with helium gas fueling [1].
Figure 9 shows the profiles of $T_{\mathrm{i}}, T_{\mathrm{e}}$, and $n_{\mathrm{e}}$ in this scan. The density profile remains more or less unchanged and is flat with central density $n_{\mathrm{e} 0}=7.5 \times 10^{19} \mathrm{~m}^{-3}$, whereas $T_{\mathrm{e}, 0}$ increases from 1.3 to $3 \mathrm{keV}$. The ion temperature variation is smaller and $T_{\mathrm{i}, 0}$ reduces from $1.7 \mathrm{keV}$ to $1.2 \mathrm{keV}$. In this experiment, the ambipolar radial electric field $E_{\mathrm{r}}$ is deliberately kept negative across the scan, to avoid neoclassical root transitions from electron to ion root, which occur when the field changes direction [23]. This has been achieved by operating at 

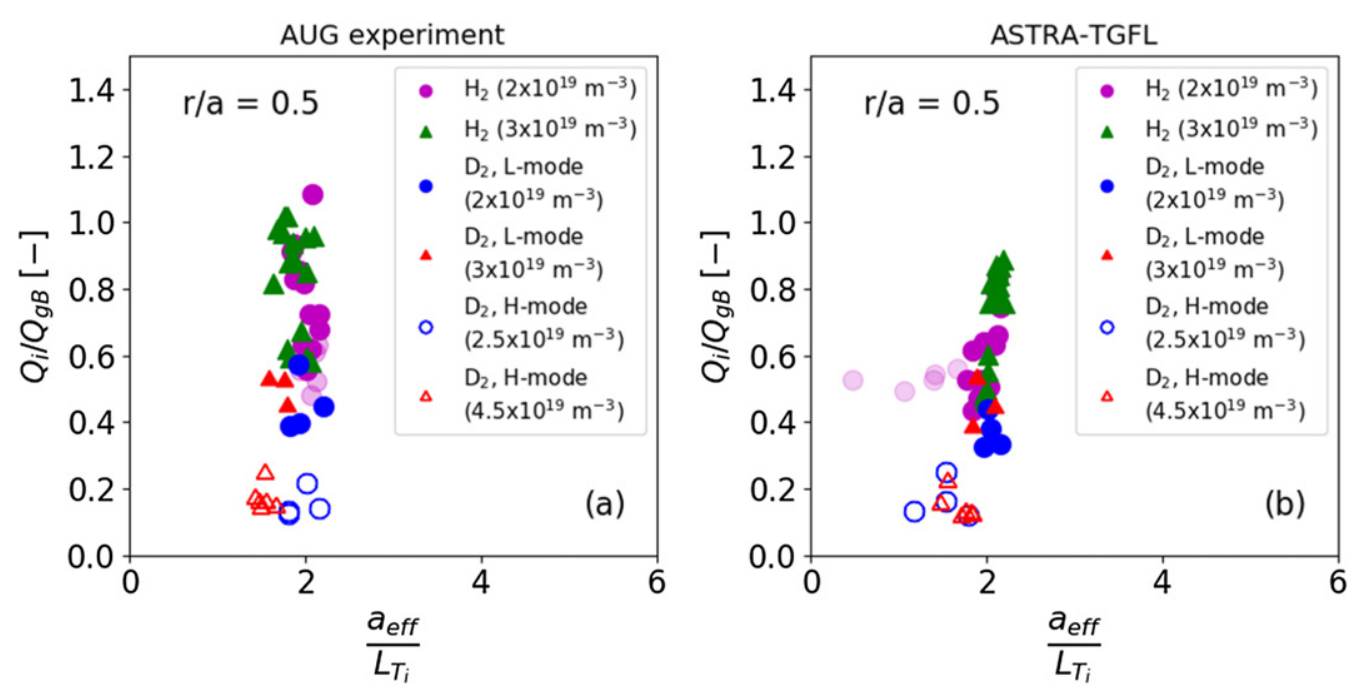

Figure 8. AUG: (a) clamping of the normalized $T_{\mathrm{i}}$ gradient at $a / L_{T_{\mathrm{i}}} \sim 2$ as a function of heat flux $Q_{\mathrm{i}} / Q_{\mathrm{gB}}$. at $r / a=0.5$. $\mathrm{H}_{2}$ and $\mathrm{D}_{2}$ plasmas clamp at similar $a / L_{T_{\mathrm{i}}}(b)$ ASTRA-TGLF simulations reproduce similar clamping effect as in the experiment. The shaded points indicate data with $T_{\mathrm{e}} / T_{\mathrm{i}}>3$, where TGLF over-predicts the clamping of $a / L_{T_{\mathrm{i}}}$.

medium to high plasma density. $E_{\mathrm{r}}$ was calculated by NTSS from the kinetic profiles in figure 9 but the profiles were independently validated by CXRS and Doppler reflectometry measurements. As a result, the calculated (NTSS) neoclassical heat fluxes $Q_{\mathrm{NC}}$ stay without the singularities that would occur at root transitions and remain well below the experimentally determined heat flux $Q_{\exp }$ (figure $9(c)$ ). The turbulent ion heat flux is determined by the difference $Q_{\mathrm{i}, \text { turb }}=Q_{\mathrm{i}, \text { exp }}-Q_{\mathrm{i}, \mathrm{NC}}$ and $A \cdot Q_{\mathrm{i} \text {,turb }}$ varies from $0.1 \mathrm{MW}$ to $0.8 \mathrm{MW}$ from the lowest to the highest ECRH power level. Figure $9(d)$ shows that the $T_{\mathrm{i}}$ profile shape changes over the power scan: outside $r / a \sim 0.5$ the normalized ion temperature gradient $a / L_{T_{\mathrm{i}}}$ increases whereas inside this radius it decreases with increasing ion heat flux $Q_{\text {i,turb. }}$

This is clearly seen in figure 10 for two selected radii showing the inverse response of the turbulent heat flux $Q_{\mathrm{i} \text {,turb }}$ to $a / L_{T_{\mathrm{i}}}$ at $r / a=0.3$ and $r / a=0.6$ respectively. To be able to compare the experimental results with gyrokinetic symulations, the turbulent heat flux is again normalized to the gyro-Bohm heat flux as $Q_{\mathrm{i}, \text { turb }} / Q_{\mathrm{gB}}$ in figures $10(b)$ and $(d)$. The strong $T_{\mathrm{i}}$ dependence of the gyro-Bohm normalization again compresses the range covered, and for the highest heat flux actually leads to a reduction of the gyro-Bohm normalized heat flux compared to the second highest ECRH power point in figure 10. Nevertheless it should be pointed out that in this experiment $Q_{\mathrm{i}, \text { turb }} / Q_{\mathrm{gB}}$ could be increased as $P_{\mathrm{ECRH}}$ was increased to allow testing the effect of $T_{\mathrm{e}} / T_{\mathrm{i}}$ on the local turbulence. This was not possible for the AUG experiment in the previous section.

At the small radius $r / a=0.3$ it seems rather counter intuitive that the reduction in normalized gradient length $a / L_{T_{\mathrm{i}}}$ is due to an apparent 'negative' ion temperature profile stiffness. Clearly a strong transport driver is also here at play that reduces the local normalized gradient $a / L_{T_{\mathrm{i}}}$, despite an increase in heat flux.

Linear gyro kinetic simulations have been performed using the GENE code [24] to help identify the type of turbulence at work. As an example we show here the analysis using the kinetic profiles in figure 9 for the highest ECRH power level of $P_{\mathrm{ECRH}}=3.9 \mathrm{MW}$. Figure 11 shows four simulation scenarios to identify different turbulence types: ITG turbulence using only the $T_{\mathrm{i}}$ profile $\left(a / L_{T_{\mathrm{i}}} \neq 0\right)$, TEM using only the $n_{\mathrm{e}}$ profile $\left(a / L_{n} \neq 0\right)$, ETG using only the $T_{\mathrm{e}}$ profile $\left(a / L_{T_{\mathrm{e}}} \neq 0\right)$, and mixed turbulence using all profiles $\left(a / L_{T_{\mathrm{i}}} \neq 0, a / L_{\mathrm{n}} \neq 0\right.$, $\left.a / L_{T_{\mathrm{e}}} \neq 0\right)$.

For the inner radial $r / a=0.3$ (figures $11(a)$ and $(b)$ ) both ITG and/or mixed ITG/TEM are dominant instabilities at ion scales (positive real frequency in the 'all'-curve around $k_{\perp} \rho \sim 1$ ). Negative real frequency of the pure 'ETG'-curve and the ETG trend in the 'all'-curve suggests an ETG dominant instability at intermediate scales $k_{\perp} \rho>1.5$. A quasilinear argument based on diffusivity $D \sim \gamma / k_{\perp}{ }^{2}$ together with an ITG/ITG-TEM instability around $k_{\perp} \rho \sim 1$ and a dominant ETG at shorter scales suggest a dominant ITG/ITG-TEM mixed instability in the total ion transport.

At $r / a=0.6$ (figures $11(c)$ and $(d)$ ) strong dominant ITG activity at ion and intermediate scales is found (positive frequencies in 'all'-curve up to $k_{\perp} \rho=3.5$ ). TEM activity is also present but the 'all'-curves follow a similar trend to the pure 'ITG'-curves. ETG turbulence seems to become dominant for $k_{\perp} \rho>3.5$. A quasi-linear argument based on diffusivity $D \sim \gamma / k_{\perp}^{2}$ together with a strong ITG instability around for $k_{\perp} \rho \sim 1$ suggests a dominant ITG instability in the total ion transport.

Across the entire radial cross section (figures 11(e) and $(f)$ ), the 'all'-curve clearly follows the pure-ITG trend reinforcing the observation that ITG is the dominant instability at ion and intermediate scales and that ion heat transport in these plasmas is driven by ITG turbulence.

An illustration of the processes that contribute to the ion temperature clamping in ECRH heated plasmas in W7-X, is then given by a series of linear gyrokinetic simulations with 

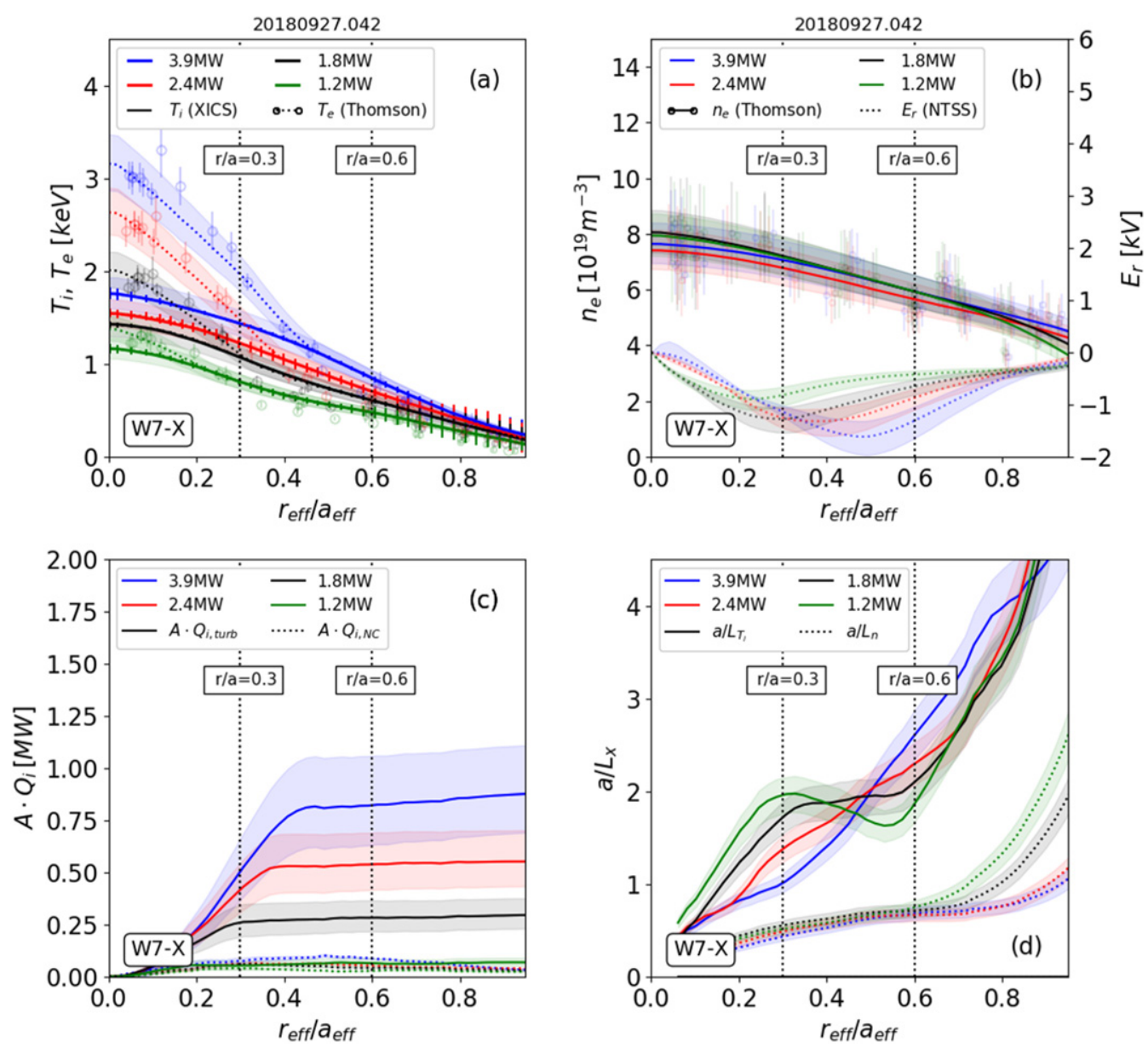

Figure 9. W7-X: profiles for the ECRH power scan $\left(P_{\mathrm{ECRH}}=1.2-3.9 \mathrm{MW}\right.$ from green to blue) in the Helium fueled plasma: $(a) T_{\mathrm{i}}$ (full) by x-ray imaging spectroscopy (XICS) and $T_{\mathrm{e}}$ (dotted) using Thomson scattering. (b) $n_{\mathrm{e}}$ (full) by Thomson scattering, and ambipolar radial electric field $E_{\mathrm{r}}$ (dotted) as calculated by NTSS $(c)$ Turbulent and neoclassical heat fluxes for the ions (see text). (d) Inverse gradient lengths for $T_{\mathrm{i}}$ (full) and $n_{\mathrm{e}}$ (dots).

the GENE code in figure 12, following [17], and following up on discussions in [25]. In these simulations, the flux-tube calculations assuming kinetic electrons and ions, are conducted for the 'standard' magnetic configuration with the normalized density gradient fixed at $a / L_{n}=0.5$, similar to the experiment (figure $9(d)$ ). To suppress ETG in this set of simulations at hand, the electron temperature gradient is set at $a / L_{T_{\mathrm{e}}}=0$. The $T_{\mathrm{e}} / T_{\mathrm{i}}$ effect on profile stiffness means that with enhancing the ratio of $T_{\mathrm{e}} / T_{\mathrm{i}}$ the ITG growth rates for a given $a / L_{T_{\mathrm{i}}}$ rise and the profile stiffness is enhanced. The exacerbation of ITG turbulence by the ratio of $T_{\mathrm{e}} / T_{\mathrm{i}}$ may indeed be responsible for the negative response of $a / L_{T_{\mathrm{i}}}$ on $Q_{\mathrm{i} \text {,turb }}$ at $\mathrm{r} / a=0.3$, whereas at $r / a=0.6$ with $T_{\mathrm{e}} / T_{\mathrm{i}}=1$ a positive response is expected. Indeed in the experiment the ratio $T_{\mathrm{e}} / T_{\mathrm{i}}$ increases from 1 to 1.5 with increasing $P_{\mathrm{ECRH}}$ at $r / a=0.3$. Further out at $r / a=0.6$ the ratio $T_{\mathrm{e}} / T_{\mathrm{i}} \sim 1$ remains constant for all four power steps. The quantitative comparison of the degree of stiffness observed in the experiment and more integrated (nonlinear) girokinetic simulations is an ongoing study.

\section{Discussion}

In both AUG and W7-X devices, ECRH heated plasmas feature ion temperature clamping where the central ion temperature does not rise above at $T_{\mathrm{i}} \sim 1.5 \mathrm{keV}$. This clamping can be the result of a combination of effects: (I) the limited and broad ion heating from power transfer from electrons in ECRH heated plasmas. (II) The expected increase of heat transport according to the gyro-Bohm scaling and (III) the exacerbated turbulent heat transport due the effect of increasing $T_{\mathrm{e}} / T_{\mathrm{i}}$ ratio on ITG or TEM turbulence. In the AUG ECRH plasmas effects (I) and (II) could be demonstrated. Effect (III), although demonstrated for AUG mixed NBI and ECRH heated plasmas in [3], could not directly be derived from the pure ECRH experiment presented here. In the W7-X study, indications that all three effects (I)-(III) contribute to $T_{\mathrm{i}}$ clamping were provided.

Mitigating the effect of ion temperature clamping in electron-heated plasmas in both tokamak and stellarator devices requires that additional turbulence suppression 

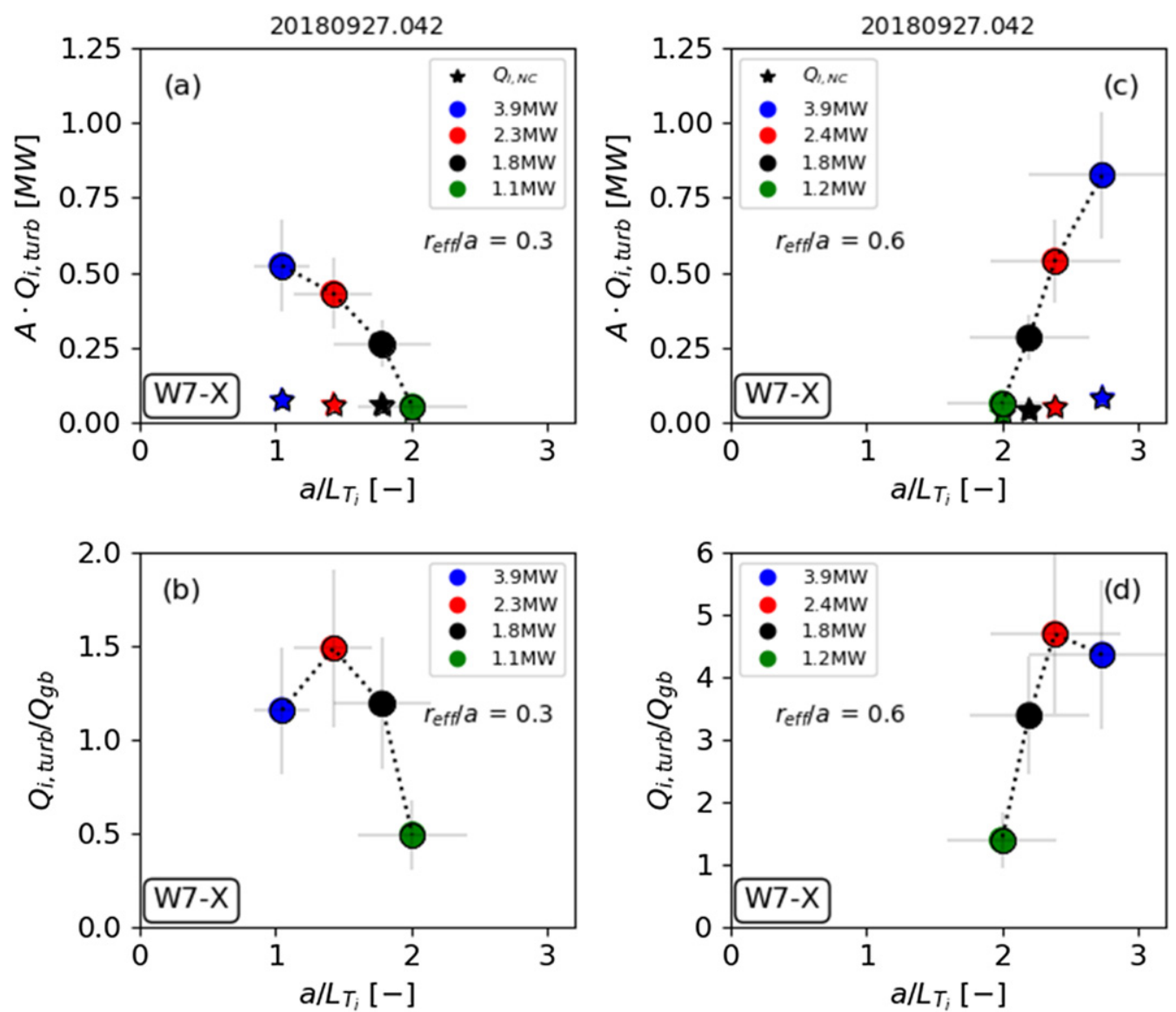

Figure 10. W7-X: response of normalized ion temperature gradient $a / L_{T_{\mathrm{i}}}$ on turbulent ion heat flux $Q_{\mathrm{i} \text {,turb }}$ in MW, as well as turbulent ion heat flux $Q_{\mathrm{i}, \text { turb }}$ in gyro-Bohm units (see text). For $(a) \&(b) r / a=0.3$ and $(c) \&(d) r / a=0.6$.

mechanisms are found. In a tokamak, this problem may be ameliorated by means of the edge pedestal in $H$-mode plasmas. In absence of an $H$-mode with strong pedestal temperatures, other turbulence suppression mechanisms are required.

In W7-X, favorable stability properties have been demonstrated, that may help improve the core confinement by a reduction of the core turbulent transport. In [7] electrostatic linear gyrokinetic simulations are conducted to scan the relevant parameter space for the W7-X standard configurations, resulting in stability maps that account for the key microinstabilities thought to drive turbulent transport. These maps exhibits a 'stability valley' in the region where $a / L_{n} \sim a / L_{T_{\mathrm{i}}}$. In this valley, the electrostatic instabilities are partly suppressed thanks to beneficial geometry property of the W7-X field [7]. Figure 13 shows examples where plasmas are analyzed to have accessed the stability valley. For comparison the figure shows the $T_{\mathrm{i}}$ clamping in (1) the helium and (2) its reference hydrogen fueled ECRH plasmas discussed in [1]. The $T_{\mathrm{i}}$-clamping was broken in (3) plasmas shortly after a boronization at low input power and increased edge density gradients in (4) plasmas with boron power dropping [26], also providing enhanced edge density gradients. (5) The highest $T_{\mathrm{i}} \sim 3 \mathrm{keV}$ has been obtained in pellet experiments with strong density peaking, leading to ITG turbulence suppression in the post pellet phase allowing strongly enhanced $a / L_{T_{\mathrm{i}}} \sim \mathrm{a} / L_{n}>3$ [9-11]. Unfortunately, this high $T_{\mathrm{i}}$ phase was transient and $T_{\mathrm{i}}$ reduced again after the density gradient was depleted. In W7-X, new scenario tools such as a continuous ice-pellet injector, cryo-gas pumping, core particle fueling and ion heating through neutral beam injection will be exploited to study higher performance plasmas with suppressed core turbulence. 

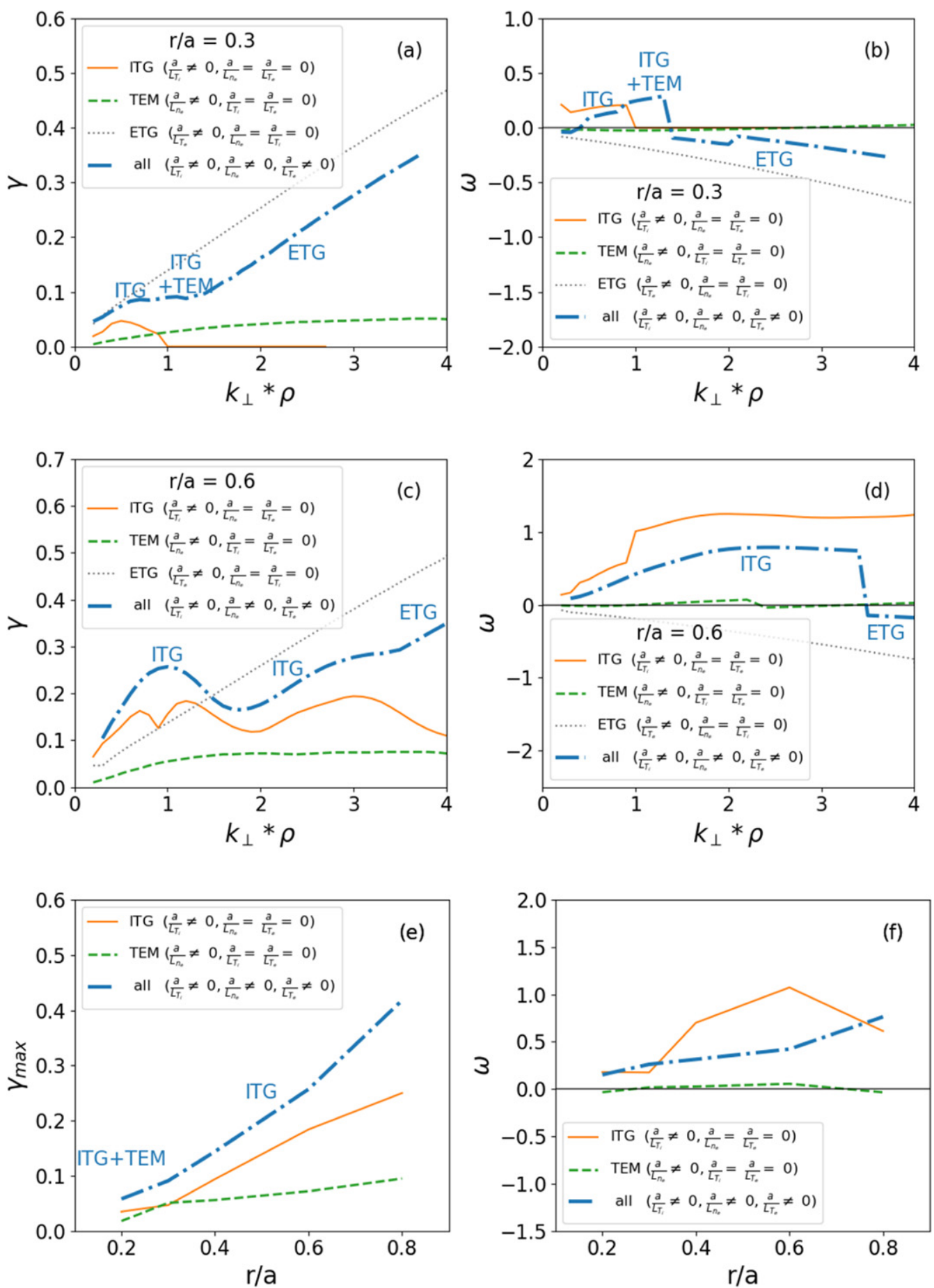

Figure 11. W7-X: linear gyro kinetic simulations using the experimental profiles for the $P_{\mathrm{ECRH}}=3.9 \mathrm{MW}$ case of figure 9 for four scenarios: (all) the actual discharge scenario with all profile gradients included, (ITG) pure ITG scenario with only $a / L_{T_{\mathrm{i}}}$ included, (TEM) pure TEM scenario with only $a / L_{n}$ included and (ETG) pure ETG scenario (only $a / L_{T_{\mathrm{e}}}$ included). The figures show the growth rate $(a),(c)$ and real frequency $(b),(d)$ spectrum for $r / a=0.3$ and $r / a=0.6$. respectively and $(e)$ Radial profiles of the maximum growth rate at ion scales. And $(f)$ Radial profiles of the real frequency at ion scales. 


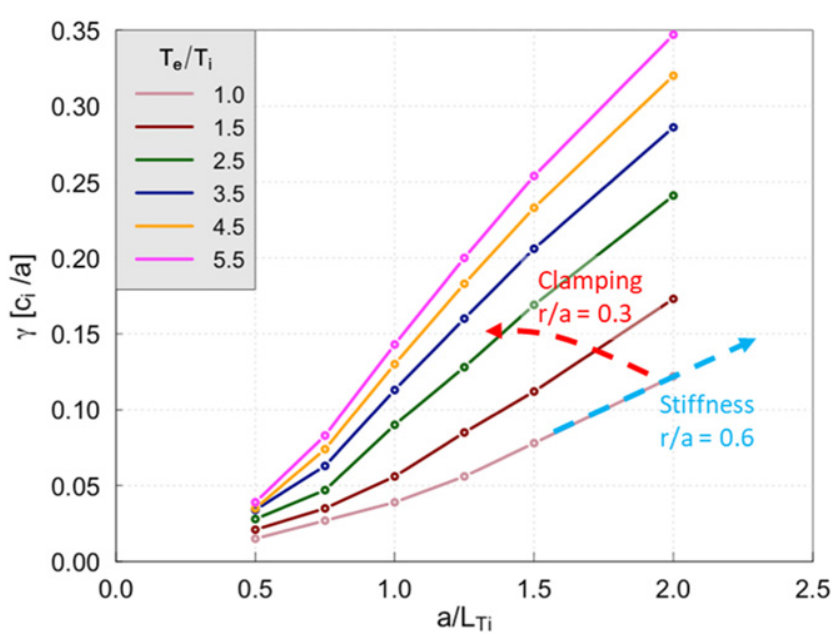

Figure 12. W7-X Illustration of the $T_{\mathrm{e}} / T_{\mathrm{i}}$ effect on ITG turbulence using linear flux tube averaged gyrokinetic simulations, showing the growth rate $\gamma$ of the most dominant ITG mode as a function of normalized ion temperature gradient $a / L_{T_{\mathrm{i}}}$. The red dashed line shows the increasing degree of stiffness as the ratio of $T_{\mathrm{e}} / T_{\mathrm{i}}$ is increased. This is mechanism may represent the observations of 'negative' stiffness at $r / a=0.3$ in figure 10 , whereas the cyan dashed line shows the classical profile stiffness that may represent the $a / L_{T_{\mathrm{i}}}$ changes at $r / a=0.6$

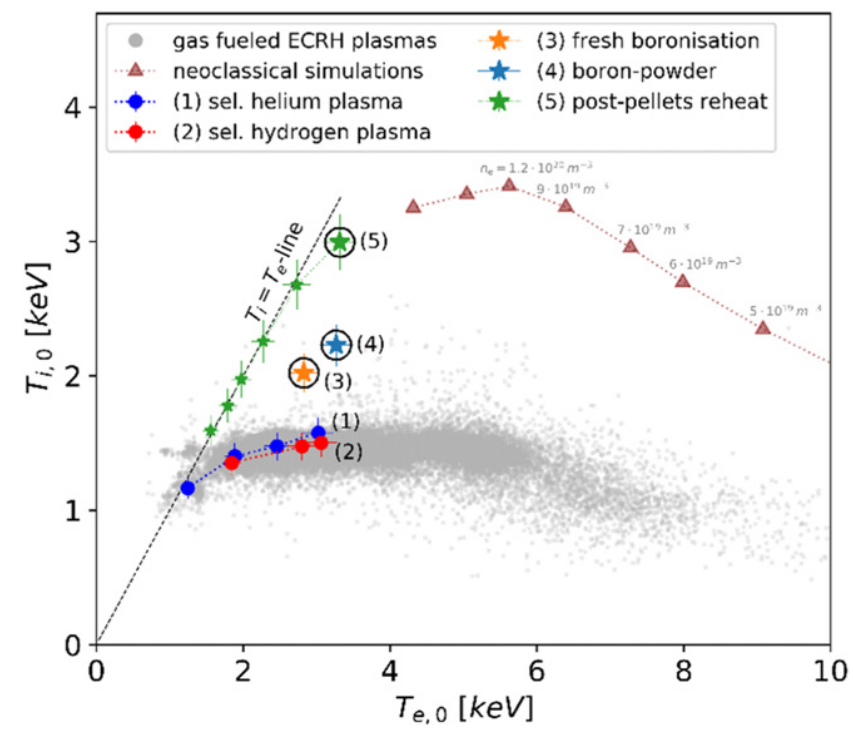

Figure 13. W7-X: mitigating ion temperature clamping in W7-X with example plasmas showing the back ground data as in figure 1 as well as the $T_{\mathrm{i}}$-clamping in (1) the ECRH helium fueled plasma in figure 9 and its (2) hydrogen reference. The example plasmas (3)-(5) are analyzed to have approached or accessed the stability valley (see main text) and hence elevated $T_{\mathrm{i}}$ could be achieved with transiently the record $T_{\mathrm{i}, 0}=3 \mathrm{keV}$.

\section{Acknowledgments}

This work has been carried out within the framework of the EUROfusion Consortium and has received funding from the Euratom research and training programme 2014-2018 and 2019-2020 under Grant Agreement No. 633053. The views and opinions expressed herein do not necessarily reflect those of the European Commission.

\section{ORCID iDs}

M.N.A. Beurskens (D) https://orcid.org/0000-0002-3354-0279

C. Angioni (D) https://orcid.org/0000-0003-0270-9630

S. A. Bozhenkov (D) https://orcid.org/0000-0003-4289-3532

P. Xanthopoulos (iD https://orcid.org/0000-0003-3545-4822

J.A. Alcusón (iD https://orcid.org/0000-0001-5492-7432

C. Beidler (D) https://orcid.org/0000-0002-4395-239X

M. Jakubowski (i) https://orcid.org/0000-0002-6557-3497

A. Langenberg (D) https://orcid.org/0000-0002-2107-5488

S. Lazerson (1) https://orcid.org/0000-0001-8002-0121

N. Pablant (D) https://orcid.org/0000-0001-6617-8459

D. Zhang (i) https://orcid.org/0000-0002-5800-4907

R.C. Wolf (D) https://orcid.org/0000-0002-2606-5289

\section{References}

[1] Beurskens M.N.A. et al 2021 Nucl. Fusion 61116072

[2] Garbet X. et al 2004 Plasma Phys. Control. Fusion 461351

[3] Ryter F. et al 2019 Nucl. Fusion 59096052

[4] Proll J.H.E., Helander P., Connor J.W. and Plunk G.G. 2012 Phys. Rev. Lett. 108245002

[5] Helander P., Bird T., Jenko F., Kleiber R., Plunk G.G., Proll J.H.E., Riemann J. and Xanthopoulos P. 2015 Nucl. Fusion 55053030

[6] Plunk G.G., Connor J.W. and Helander P. 2017 J. Plasma Phys. 83715830404

[7] Alcusón J.A., Xanthopoulos P., Plunk G.G., Helander P., Wilms F., Turkin Y., Stechow A.v. and Grulke O. 2020 Plasma Phys. Control. Fusion 62035005

[8] Xanthopoulos P. et al 2020 Phys. Rev. Lett. 125075001

[9] Bozhenkov S.A. et al 2020 Nucl. Fusion 60066011

[10] Baldzuhn J. et al 2020 Plasma Phys. Control. Fusion 62055012

[11] Beidler C.D. et al 2021 Nature 596 221-6

[12] Turkin Y., Maassberg H., Beidler C.D., Geiger J. and Marushchenko N.B. 2006 Fusion Sci. Technol. 50 387-94

[13] Turkin Y., Beidler C.D., Maaßberg H., Murakami S., Tribaldos V. and Wakasa A. 2011 Phys. Plasmas 18022505

[14] Ryter F. et al 2009 Nucl. Fusion 49062003

[15] Wesson J. 2003 Tokamaks (Oxford: Clarendon)

[16] Xanthopoulos P. et al 2014 Phys. Rev. Lett. 113155001

[17] Zocco A., Xanthopoulos P., Doerk H., Connor J.W. and Helander P. 2018 J. Plasma Phys. 84715840101

[18] Fable E. et al 2013 Plasma Phys. Control. Fusion 55124028

[19] Staebler G.M., Candy J., Howard N.T. and Holland C. 2016 Phys. Plasmas 23062518

[20] Kiefer C.K., Angioni C., Tardini G., Bonanomi N., Geiger B., Mantica P., Pütterich T., Fable E. and Schneider P.A. 2021 Nucl. Fusion 61066035

[21] Staebler G.M., Candy J., Belli E.A., Kinsey J.E., Bonanomi N. and Patel B. 2021 Plasma Phys. Control. Fusion 63 015013

[22] Casati A., Bourdelle C., Garbet X. and Imbeaux F. 2008 Phys. Plasmas 15042310

[23] Maaßberg H., Beidler C.D. and Simmet E.E. 1999 Plasma Phys. Control. Fusion 41 1135-53

[24] Jenko F., Dorland W., Kotschenreuther M. and Rogers B.N. 2000 Phys. Plasmas 71904

[25] Wegner Th. et al 2020 Nucl. Fusion 60124004

[26] Lunsford R. et al 2021 Phys. Plasmas 28082506 